\title{
Novel reconstruction of a vascular aneurysm in Marfan syndrome
}

\author{
Xun Yuan, Andreas Mitsis, Christoph A. Nienaber \\ Cardiology and Aortic Centre, Royal Brompton \& Harefield NHS Trust and Imperial College, London, UK \\ Correspondence to: Prof. Christoph A. Nienaber, MD, PhD. Cardiology and Aortic Centre, Royal Brompton \& Harefield NHS Trust and Imperial \\ College, Sydney Street, London, SW3 6NP, UK. Email: C.Nienaber@rbht.nhs.uk.
}

Received: 23 January 2018; Accepted: 12 March 2018; Published: 03 April 2018.

doi: 10.21037 /jovs.2018.03.12

View this article at: http://dx.doi.org/10.21037/jovs.2018.03.12

The role of endovascular procedures in Marfan syndrome (MFS) remains an issue of debate. In principle, endovascular techniques are not intended for the treatment of aortic or vascular conditions in patients with genetic connective tissue disease. Despite the highly restrictive use of endovascular technology in genetically linked conditions, more than 69 cases have been published (1), with the impression that endovascular treatment may have a place as life-saving bridging procedure until a definitive open repair can be performed, while elective endovascular management is not established.

We report here the elective endovascular management of a subclavian aneurysm of a 46-year-old female Marfan patient after multiple surgeries for vascular and aortic manifestations. In 2005, she had a Crawford II repair for a descending aortic dissection followed by a valvesparing aortic root replacement in 2008 for ascending aortic dissection, and bilateral mastectomy for breast cancer (BRCA2 mutation) in 2007. In 2016, a routine computed tomography (CT) angiogram revealed a new circumscript aneurysm $(4.1 \times 3.3 \mathrm{~cm})$ of the left subclavian artery (LSA) with retro-clavicular intrathoracic location. A pulsatile sensation in the left supra-clavicular fossa and increasing hoarseness were noted, confirmed by palpable swelling. The patient was initially offered surgical resection, however, with critically reduced lung function, was found at high risk and opted for an endovascular solution. Thus, a highly flexible self-expanding stent-graft (Viabahn ${ }^{\circledR}$ $9 \times 100 \mathrm{~mm}$ ) was placed across the aneurysm with about $3 \mathrm{~cm}$ of landing zone on either side, leaving left vertebral and mammary artery unobstructed. For this percutaneous strategy, a short $9 \mathrm{~F}$ sheath was advanced into the left brachial artery. Under heparin, a " 0.035 " guide-wire was navigated across the aneurysm to reach the ascending aorta, followed by a Viabahn ${ }^{\circledR}$ stent-graft $(9 \times 100 \mathrm{~mm})$ along the wire and precisely deployed to exclude the aneurysm. A completion angiogram confirmed correct positioning and absence of endoleak; the brachial artery access was closed by manual compression for 30 minutes followed by a TRband $^{\circledR}$ for 3 hours. Before hospital discharge the next day, a CT angiogram demonstrated precise deployment and no compromise to vital side branches and complete exclusion of the aneurysm. The patient went home on combination medication of Aspirin $75 \mathrm{mg}$, Bisoprolol $5 \mathrm{mg}$, and Losartan $50 \mathrm{mg}$, and was followed for 1-year; at 1-year follow-up, a CT scan confirmed no endoleak, and eventually complete abolition of the vascular aneurysm (Figure 1). As durability of an endovascular procedure in MFS patients is still debatable, the patient will be followed at annual intervals.

\section{Discussion}

Even in patients with MFS, percutaneous "neo-branching" can provide a satisfactory solution with safe and sustained exclusion of an aneurysm by anchoring a graft snuggly within target vessel (2). The case represents the departure from the conventional open approach of resecting a subclavian artery aneurysm and confirms the safe and lasting result of an endovascular strategy in MFS. Particularly in cases with multiple previous thoracotomies and major surgery, a minimally invasive procedure may be the best option regardless of the background of hereditary condition such as Marfan. Self-expanding highly flexible stentgrafts could be the best option in such vascular pathologies because they are less prone to deformation with movement of the shoulder joint; hence, the heparin-coated Viabahn ${ }^{\circledR}$ stent-graft is highly flexible allowing perfect apposition to the tortuous anatomy in the subclavian region $(3,4)$. Finally, with follow-up over 18 months, no dislocation or graft failure has been noted. 

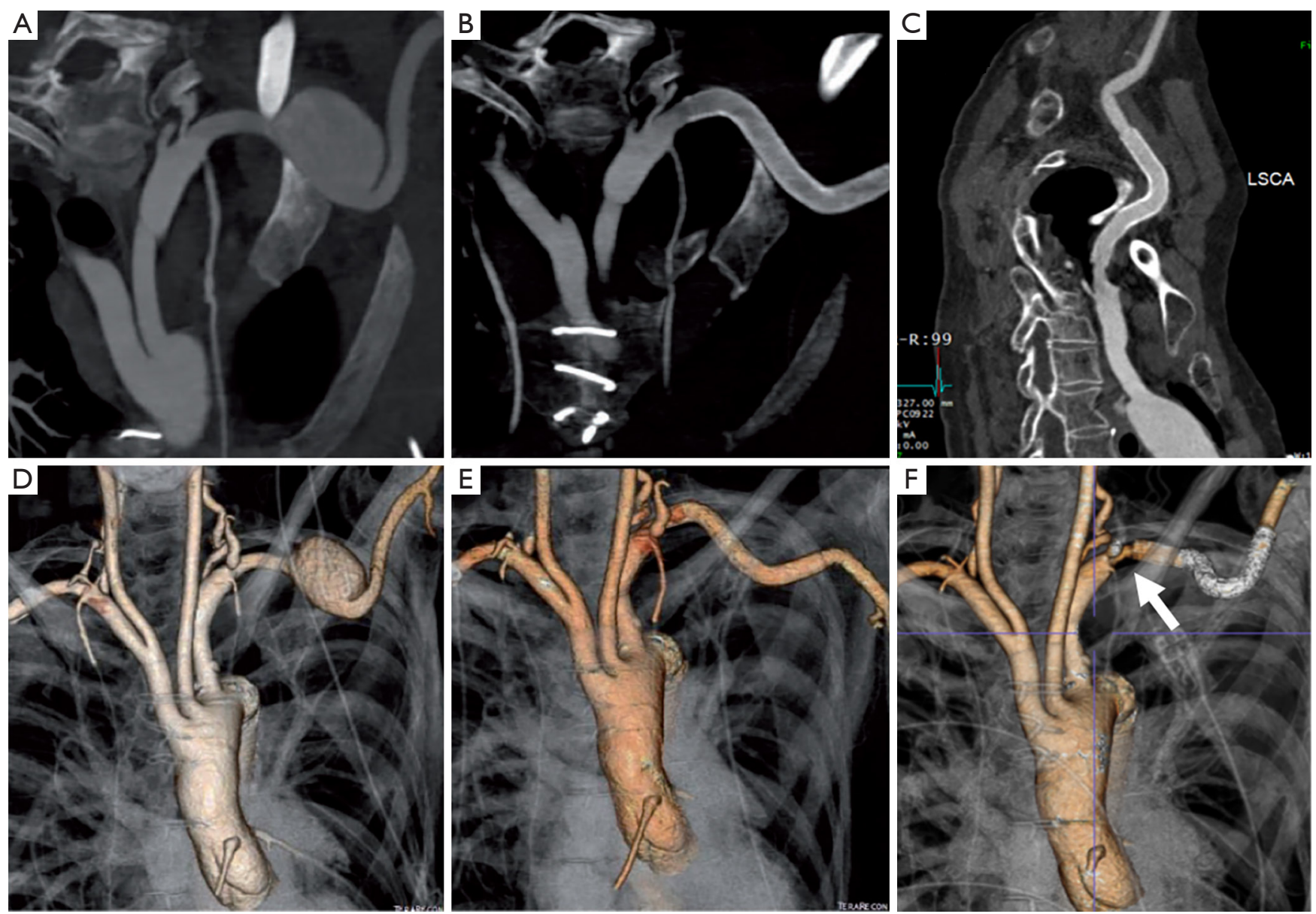

Figure $12 \mathrm{D}$ and 3D volume rendered computed tomography angiogram demonstrates. (A,D) Pre-procedure, Circumscript LSA aneurysm measuring $(4.1 \times 3.3 \mathrm{~cm})$; (B,E) post-procedure; before hospital discharge, the LSA aneurysm was completely excluded after deployment of Viabahn ${ }^{\circledR}$ stent-graft $(9 \times 100 \mathrm{~mm}) ;(\mathrm{C}, \mathrm{F})$ at 1-year follow-up, a CT scan showed the LSA stent-graft remains widely patent with no evidence of the previous aneurysm, immediately proximal to the stent and distal to the left vertebral origin, there is a moderate concentring narrowing with an estimated stenosis $30 \%$ (arrow).

Although the number of patients with MFS subjected to endovascular treatment is still small, the reported results are indeed encouraging with very low mortality. Conversely, the rate of reinterventions for primary and/or secondary endoleak (with lack of remodelling) is still high. Dong et al. (5) reported a frequent complication after stentgraft implantation in MFS's retrograde aortic dissection (rTAD). The European registry on endovascular aortic repair complication (EUREC) could confirm the higher incidence of retrograde dissection with $8.4 \%$ in the case of MFS (6). Thus, TEVAR in MFS patients should only be considered if devices are highly conformable and flexible and thereby atraumatic. Stent graft-induced new entry (SINE) tears can occur and are more likely to occur in
MFS. Mortality of SINE is significant and was quoted at around $30 \%$ (7). In the setting of MFS, the incidence of SINE is higher at $33 \%$ compared to $3 \%$ in non-Marfan patients (7). Thus, patients with hereditary aortic condition are currently not yet considered good candidates for endovascular management. In addition, recent consensus paper on open and endovascular management in patients with thoracic aortic disease obtains from recommending endovascular procedures in Marfan patients unless conventional open surgery incurs some prohibitive risk $(8,9)$. Endovascular procedures may be accepted as bailout procedures or as a concept of bridging to classic open surgical repair, or as a procedure after aortic repair when landing zones constitute surgically implanted prosthetic 
grafts. Recent European guidelines on the management of aortic diseases corroborate that classic open surgery should be offered as first-line therapy (class IIa, level of evidence C), except in an emergency setting to stabilise or bridge to definitive surgical repair (10). On aggregate, endovascular management is becoming an option for even MFS patients with a prohibitively high surgical risk; in selected cases can be safely placed for instance in the presence of a proximal or distal prosthetic graft in place. Moreover, endovascular management can also be an option of emergency situations like complicated type B acute aortic dissections and ruptured aneurysms when surgical outcomes are sobering. Regardless of treatment, close clinical and imaging surveillance appears to be mandatory to monitor outcomes and to identify early stent-graft related complications or disease progression in every case.

\section{Conclusions}

In patients with hereditary aortic conditions management of vascular pathology is highly complex; modern endovascular management is now providing the option not only as an adjunct or bridge to later elective surgical repair but also to provide a definitive solution both in an emergency and elective setting.

\section{Acknowledgements}

None.

\section{Footnote}

Conflicts of Interest: The authors have no conflicts of interest to declare.

\section{References}

1. Parisi R, Secco GG, Di Eusanio M, et al. Endovascular

doi: 10.21037/jovs.2018.03.12

Cite this article as: Yuan X, Mitsis A, Nienaber CA. Novel reconstruction of a vascular aneurysm in Marfan syndrome. J Vis Surg 2018;4:67.
Repair of Aortic Dissection in Marfan Syndrome: Current Status and Future Perspectives. Diseases 2015;3:159-66.

2. Vandormael IL, Salmasi MY, Yeh JS, et al. Endovascular "neobranching" to manage acute aortic syndrome. Catheter Cardiovasc Interv 2017;90:298-302.

3. Kasirajan K, Matteson B, Marek JM, et al. Covered stents for true subclavian aneurysms in patients with degenerative connective tissue disorders. J Endovasc Ther 2003;10:647-52 .

4. Tassiopoulos AK, Nadalin BA, Labropoulos N, et al. Endovascular repair of a symptomatic subclavian artery aneurysm in a patient with Marfan syndrome: a case report. Vasc Endovascular Surg 2006;40:409-13.

5. Dong ZH, Fu WG, Wang YQ, et al. Retrograde type A aortic dissection after endovascular stent graft placement for treatment of type B dissection. Circulation 2009;119:735-41.

6. Eggebrecht $\mathrm{H}$, Thompson $\mathrm{M}$, Rousseau $\mathrm{H}$, et al. Retrograde ascending aortic dissection during or after thoracic aortic stent graft placement: insight from the European registry on endovascular aortic repair complications. Circulation 2009;120:S276-81.

7. Dong Z, Fu W, Wang Y, et al. Stent graft-induced new entry after endovascular repair for Stanford type B aortic dissection. J Vasc Surg 2010;52:1450-7.

8. Svensson LG, Kouchoukos NT, Miller DC, et al. Expert consensus document on the treatment of descending thoracic aortic disease using endovascular stent-grafts. Ann Thorac Surg 2008;85:S1-41.

9. Fattori R, Cao P, De Rango P, et al. Interdisciplinary expert consensus document on management of type $B$ aortic dissection. J Am Coll Cardiol 2013;61:1661-78.

10. Erbel R, Aboyans V, Boileau C, et al. 2014 ESC Guidelines on the diagnosis and treatment of aortic diseases: Document covering acute and chronic aortic diseases of the thoracic and abdominal aorta of the adult. The Task Force for the Diagnosis and Treatment of Aortic Diseases of the European Society of Cardiology (ESC). Eur Heart J 2014;35:2873-926. 